\title{
Awareness and Access to Therapy in Hepatitis C Virus Infected Patients, Key Barriers to Eliminate the Virus?
}

\author{
Do Young Kim \\ Department of Internal Medicine, Yonsei University College of Medicine, Seoul, Korea
}

See "Epidemiological and Clinical Characteristics of Hepatitis C Virus Infection in South Korea from 2007 to 2017: A Prospective Multicenter Cohort Study" by Joon Yeul Nam, et al. on page 207, Vol. 14, No. 2, 2020

Antiviral therapy with direct-acting antiviral (DAA) for patients infected by hepatitis $\mathrm{C}$ virus (HCV) has made a remarkable progress, enabling more patients eligible for treatment compared to interferon era. Indeed, DAA treatment with high efficacy and safety is an important major component of global strategy to eliminate HCV infection until 2030, proposed by the World Health Organization (WHO). ${ }^{1}$ The epidemiological and clinical characteristics related to HCV infection largely differ among regions and countries. Even in a region or country, these characteristics might be different between two time periods which are divided by introduction of DAA. Thus, it is relevant to figure out which characteristics are clinically significant in post-DAA period compared with pre-DAA period to help implement an effective program for HCV elimination.

In this issue, Nam et al. ${ }^{2}$ investigated the changing epidemiological and clinical characteristics of HCV infection in South Korea between 2007 and 2017. A total of 2,758 patients with HCV viremia were prospectively enrolled at seven tertiary hospitals. Apart from various data collected from medical records, a survey was conducted using patient questionnaire to reveal risk factors for HCV infection. The overall characteristics of patients including mean age, disease severity ranging from chronic hepatitis, cirrhosis to hepatocellular carcinoma (HCC) and HCV genotypes were not much different from those previously reported. ${ }^{3}$ The major risk factors associated with HCV infection in South Korea were known to be multiple sexual partners, tattooing, history of transfusion, acupuncture and needle stick injuries. ${ }^{4}$ This article also confirms that multiple factors such as tattooing, piercing, blood transfusion and needle stick injuries contribute to HCV infection in South Korea. ${ }^{2}$ There was an epidemiological difference between males and females, showing a higher frequencies of intravenous drug use experiences, needle stick injuries and multiple sexual partners in males. Whereas such risk factors as history of transfusion, tattooing, piercing and acupuncture were more closely related to HCV infection in females, suggesting different risk factors by gender depend on the behavioral characteristics and degree of risk exposure.

Several clinical characteristics differed between pre-DAA (2007 to 2014) and post-DAA (2015 to 2017) period. The mean age of patients recently enrolled (post-DAA period) was higher than those enrolled at pre-DAA period (60.6 years vs 56.15 years, $\mathrm{p}<0.001)$. Compared to pre-DAA period, there were more male patients, less patients with education above high-school level, more patients with HCC or cardiovascular disease in postDAA period. This observation might be partly explained by older age of patients in recent period.

Since introduction of DAA in early 2000, treatment paradigm of HCV infection has been rapidly and dramatically evolved. A higher sustained virological response (SVR) rates, shorter treatment duration, better safety and tolerability resulted in higher treatment uptake rates at least in a special patient population. ${ }^{5}$ In a modeling study, combination of higher SVR rates, improved HCV diagnosis and higher treatment uptake rates was found to achieve HCV elimination until 2030 in South Korea. ${ }^{6}$ Then, are treatment uptake rates actually increasing in Korea? In this article, the overall treatment uptake rates were 52.8\% and 56.1\% in pre-DAA and post-DAA period, respectively. ${ }^{2}$ The reasons why there is not apparent increase in the treatment uptake rates

\footnotetext{
Correspondence to: Do Young Kim

Department of Internal Medicine, Yonsei University College of Medicine, 50-1 Yonsei-ro, Seodaemun-gu, Seoul 03722, Korea

Tel: +82-2-2228-1992, Fax: +82-2-393-6884, E-mail: dyk1025@yuhs.ac pISSN 1976-2283 eISSN 2005-1212 https://doi.org/10.5009/gnl20027

(a) This is an Open Access article distributed under the terms of the Creative Commons Attribution Non-Commercial License (http://creativecommons.org/licenses/by-nc/4.0) which permits unrestricted non-commercial use, distribution, and reproduction in any medium, provided the original work is properly cited.
} 
in post-DAA period compared to pre-DAA period are (1) the years from 2015 to 2017 are relatively short and early period of DAA era, (2) the efficacy of DAA regimen at that period was not accepted to be perfect by physicians, thereby postponing initiation of treatment. A subsequent study with a longer post-DAA duration is necessary to address this issue. Care of cascade consisting of screening, diagnosis, referral and treatment is mandatory to have a success in HCV elimination. According to WHO report, the treatment initiation rates in 2017 are estimated to be low globally, ranging from $2.2 \%$ in African region to $12.1 \%$ in Eastern Mediterranean region. ${ }^{7}$ Owing to different health care policy and system in each country, one unified practice strategy to enhance treatment uptake rates cannot be applied to all regions or countries. Rather, an individualized action plan based on each public health system might be more effective. For example, in South Korea, the access to HCV therapy is less limited compared to other countries because all DAAs are reimbursed by national insurance system, geographical access to hospital is easy, and patients tend to want be treated in large-volume centers. The treatment uptake rates in post-DAA period in this article, $56.1 \%$, seems to be overestimated because the participating institutions were tertiary hospitals. A nationwide treatment uptake rates would be much lower if small-volume institutions are included in the analysis. Therefore, a strategy considering this situation should be implemented in South Korea. Also, the issue of enhancing treatment uptake rates in South Korea is closed related to awareness of HCV infection by both patients and primary care physicians or doctors in department other than hepatology. Unlike in interferon era, due to short treatment duration, high efficacy and safety of DAA, hepatitis $\mathrm{C}$ became a disease which not only hepatologists but also gastroenterologist or primary care physician can manage. For the care cascade to operate effectively, government, academic society, primary care physicians and liver specialists need to play its own role in each step from screening, diagnosis, referral and to treatment. Approximately 89\% of patients were asymptomatic at infection and a half of patients showed alanine aminotransferase levels less than $40 \mathrm{IU} / \mathrm{L},{ }^{2}$ implying that diagnosis of HCV infection is difficult without screening test in general population. Because progression of HCV infection poses a significant social and economic burden, and accumulated data indicate that national anti-HCV screening once in a lifetime for general population is cost-effective, ${ }^{8,9}$ government needs to present how to eliminate HCV infection in South Korea until 2030. Academic society and liver specialists might help to make a proper HCV screening program based on experiences and evidences. Other roles of liver specialists include spreading knowledge on HCV disease into general physicians and managing "difficult-to-treat" patients.
Under well-organized health care system in both disease prevention and national insurance system in South Korea, it is time to achieve fruitful epidemiological outcomes in HCV disease as in hepatitis B virus disease for the last a few decades.

\section{CONFLICTS OF INTEREST}

No potential conflict of interest relevant to this article was reported.

\section{ORCID}

Do Young Kim https://orcid.org/0000-0002-8327-3439

\section{REFERENCES}

1. World Health Organization (WHO). Combating hepatitis B and C to reach elimination by 2030: advocacy brief [Internet]. Geneva: WHO; c2016 [cited 2019 Jul 10]. Available from: https://apps.who. int/iris/handle/10665/206453.

2. Nam JY, Jang ES, Kim YS, et al. Epidemiological and clinical characteristics of hepatitis C virus infection in South Korea from 2007 to 2017: a prospective multicenter cohort study. Gut Liver 2020;14:207-217.

3. Kim DY, Kim IH, Jeong SH, et al. A nationwide seroepidemiology of hepatitis C virus infection in South Korea. Liver Int 2013;33:586-594.

4. Sohn HS, Kim JR, Ryu SY, et al. Risk factors for hepatitis C virus (HCV) infection in Areas with a high prevalence of HCV in the Republic of Korea in 2013. Gut Liver 2016;10:126-132.

5. Boerekamps A, van den Berk GE, Lauw FN, et al. Declining hepatitis $\mathrm{C}$ virus (HCV) incidence in dutch human immunodeficiency virus-positive men who have sex with men after unrestricted access to HCV therapy. Clin Infect Dis 2018;66:1360-1365.

6. Alfaleh FZ, Nugrahini N, Matičič M, et al. Strategies to manage hepatitis $C$ virus infection disease burden - volume 3. J Viral Hepat 2015;22 Suppl 4:42-65.

7. World Health Organization (WHO). Global hepatitis report, 2017 [Internet]. Geneva: WHO; c2017 [cited 2019 Jul 10]. Available from: https://www.who.int/hepatitis/publications/global-hepatitisreport2017/en/.

8. Kim DY, Han KH, Jun B, et al. Estimating the cost-effectiveness of one-time screening and treatment for hepatitis C in Korea. PLoS One 2017;12:e0167770.

9. Kim KA, Chung W, Choi HY, Ki M, Jang ES, Jeong SH. Cost-effectiveness and health-related outcomes of screening for hepatitis C in Korean population. Liver Int 2019;39:60-69. 